\title{
A Novel ANN Fault Diagnosis System for Power Systems Using Dual GA Loops in ANN Training
}

\author{
T.S. Bi Y.X. Ni, S.M. IEEE C.M. Shen F.F. Wu, Fellow, IEEE \\ Dept. of Electrical \& Electronic Engineering \\ The University of Hong Kong \\ Hong Kong SAR
}

\begin{abstract}
Fault diagnosis is of great importance to the rapid restoration of power systems. Many techniques have been employed to solve this problem. In this paper, a novel Genetic Algorithm (GA) based neural network for fault diagnosis in power systems is suggested, which adopts three-layer feed-forward neural network. Dual GA loops are applied in order to optimize the neural network topology and the connection weights. The first GA-loop is for structure optimization and the second one for connection weight optimization. Jointly they search the global optimal neural network solution for fault diagnosis. The formulation and the corresponding computer flow chart are presented in detail in the paper. Computer test results in a test power system indicate that the proposed GAbased neural network fault diagnosis system works well and is superior as compared with the conventional Back-Propagation (BP) neural network.
\end{abstract}

Keywords Fault diagnosis, Artificial Neural Network (ANN), Genetic Algorithm (GA), power system

\section{INTRODUCTION}

To enhance service reliability and to reduce power supply interruption, rapid restoration of power system is essential. As the first step to system restoration, fault diagnosis should be implemented quickly and accurately in order to isolate the faulted elements from the rest of the system and to take proper countermeasures to recover normal power supply. However, fault diagnosis is difficult, especially for the cases with failure operations of relays and circuit breakers, or multiple faults at the same time. Therefore on-line automatic fault diagnosis is significant to the restorative operations.

Fault diagnosis is to identify the faulty elements in power systems by using the information of the current status of the protective relays and circuit breakers, which is available from SCADA systems. Many techniques have been employed to solve this problem such as expert-system-based ${ }^{[1-2]}$, fuzzyset-based ${ }^{[3-4]}$, optimization-based ${ }^{[5-6]}$, logic-based ${ }^{[7-8]}$ and artificial-neural-network-based ${ }^{[9-11]}$ approaches.

Among these approaches, Artificial Neural Network (ANN) is an active research area and has given promising results for fault diagnosis in power systems, because it has the capabilities of learning, generalization and fault tolerance. In addition, the neuron computations are parallel which make it suitable for on-line environment. However the standard Back-Propagation neural network (BP NN) ${ }^{[12-13]}$, the most widely used model in all ANNs, still suffers some imperfections. BP NN requires a pre-defined topology that the numbers of the hidden neurons and corresponding activation functions must be priori known. An optimal ANN topology could reduce the enormous search spaces in learning and then improve the system performance in terms of learning speed, accuracy and noise resistance capabilities. Until now, there is no systematic ways to select optimal ANN structure. Furthermore BP NN is a gradient search algorithm in nature, it is possible for the network solution to become trapped into one of local minimums and the discontinuous connection weights cannot be handled. These disadvantages have limited the application of the BP NN in fault diagnosis.

In recent years the use of Genetic Algorithm (GA) ${ }^{[14]}$ for the network optimization has created a large interest in $\mathrm{NN}$ area. GA is a powerful search algorithm based on the mechanism of natural selection. Unlike conventional search algorithms, it considers many points in the search space simultaneously and then reduces the chance of converging to local optima. In addition, GA could deal with any kind of problem, even ill-defined, irregular problems. Some promising results, applied GA to optimize the neural network, were reported ${ }^{(15-16)}$. But most of them only optimized the weights of the neural network and seldom mentioned the neural network topology.

In this paper, a novel Genetic Algorithm (GA) based neural network for fault diagnosis in power systems is suggested, which adopts three-layer feed-forward neural network. Dual GA loops are applied in order to optimize the neural network topology and the connection weights. The first GA-loop is for structure optimization and the second one for connection weight optimization. Jointly they search the global optimal neural network solution for fault diagnosis. The formulation and the corresponding computer flow chart are presented in detail in the paper. Computer test results in a test power system indicate that the proposed GA based neural network fault diagnosis system works well and is superior as compared with the conventional Back-Propagation (BP) neural network.

\section{GA BASED NN FAULT DIAGNOSIS SYSTEM}

To clarify the problems using $\mathrm{BP} N \mathrm{NN}$ in fault diagnosis applications, we first conduct a brief review of BP NN.

\section{A. BP neural network for fault diagnosis}

A general multi-layer feed-forward neural network is depicted in Fig. 1. The input space can be either an actual or a normalized representation. 


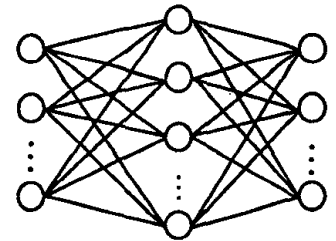

Input layer Hidden layer Output layer

Fig. 1 A multi-layer feed-forward neural network

The basic processing element is the neuron. The output of a neuron is determined as:

$$
y_{j}(p)=f\left(\sum_{i=1}^{n_{t-1}} w_{i j} y_{i}(p-1)+b(p)\right)
$$

where $y_{j}(p)$ is the output of the $j^{\text {th }}$ neuron in the $p^{\text {th }}$ layer. $w_{i j}$ is the weight from $i^{t h}$ neuron in the $(p-1)^{\text {th }}$ layer to the $j^{\text {th }}$ neuron in the $p^{\text {th }}$ layer. $n_{p-1}$ is the number of the neurons in the $(p-1)^{t h}$ layer. $b(p)$ is the bias of the $p^{t h}$ layer. $f(\cdot)$ is the activation function of the $j^{\text {th }}$ neuron, which could be a sigmoid, tansig or logsig etc. function.

A learning algorithm is needed to tune the weights and biases of the neural network to make it yield the desired outputs for the training samples. It is obvious that this is of great importance for neural network. The BP learning algorithm ${ }^{[12]}$ is based on the gradient descent algorithm, in which the performance index $E$ defined as:

$$
E=\frac{1}{2} \sum_{\mathrm{j}=1}^{\mathrm{n}_{3}}\left(d_{J}-y_{j}(p)\right)^{2}
$$

where the output layer $p$ has $n_{p}$ output neurons. $d_{j}$ and $y_{j}(p)$ are the desired and calculated output of the $j^{\text {th }}$ output neuron respectively.

Once we get the performance index, the updated value of the weights could be presented as follows:

$$
\begin{aligned}
& w_{i j}(\text { new })=w_{i j}+\Delta w_{i j} \\
& \Delta w_{i j}=-\partial E / \partial w_{i j}
\end{aligned}
$$

where $\Delta w_{i j}$ is the updated value of the $w_{i j}$.

According (3) and (4), all the weights could be updated iteratively. The learning process will be terminated until the system performance reaches a satisfactory level. The standard BP algorithm may tend to be unstable and converge slowly under certain operation conditions. Hence, several variations of BP have been proposed to overcome these demerits, such as conjugate gradient BP, Quasi-Newton BP and LevenbergMarquardt BP, etc.

During the learning process for fault diagnosis problem, there are three issues we should notice. First, it requires a predefined topology that the number of hidden neurons and corresponding activation functions must be priori known. An optimal ANN topology could reduce the enormous search spaces in learning and then improve the system performance in terms of learning speed, accuracy and noise resistance capabilities. The second issue of BP NN is that if there exist more than one local minimum points on its error surface, it is possible that the NN training is trapped into one of local minimums. Furthermore, since we use gradient descent algorithm, discontinuous connection weights cannot be handled.

In our study, we are going to solve the three problems through GA-based training rather than gradient decent training. In the training process, dual GA loops are embedded into BP NN to establish the structure and train the connection weights of neural network for fault diagnosis. The suggested GA based neural network provides a systematic way to search the global optimal neural network solution for fault diagnosis.

\section{B. GA loop for structure optimization}

The structure-GA loop is responsible for optimizing neural network structure. It starts with a set of feasible solutions to the neural network structure, referred as a population. Each solution is encoded to a chromosome of binary genes associated with the number of hidden neurons and their corresponding activation functions. For example $z=$ $\left[\begin{array}{llllll}1 & 0 & 0 & 1 & 1 & 0\end{array}\right]$ is a chromosome, in which the preceding 5 binary genes denote the number of hidden neurons and the last one gene denotes the corresponding activation function with " 0 " for "tansig" and " 1 " for "logsig".

For each chromosome or individual in the populations, its fitness is obtained by activating Levenberg-Marquardt (L-M) BP learning algorithm ${ }^{[13]}$ because the convergence process of L-M BP is stable and fast. This L-M BP operates on the ANN having the architecture defined by the corresponding genotype and will be terminated while no improvement for several iterations. The fitness function of the structure-GA loop is evaluated based on two aspects: the accuracy of the network output and the complexity of the network. The accuracy of the network $f_{l}(z)$ is defined as:

$$
f_{1}(z)=\min \left\{\frac{1}{N} \sum_{i=1}^{N}(d(t)-y(p, t))^{2}\right\}
$$

where $z$ is the chromosome of the populations. $N$ is the number of training patterns in the training sample set. $p$ denoting the output layer, so $d(t)$ and $y(p, t)$ are the desired and the calculated output of the neural network for the $t^{\text {th }}$ training sample respectively.

The complexity of the network $f_{2}(z)$ is simply defined as the number of the hidden neurons. Then the fitness function of structure-GA loop could be expressed as follows:

$$
f(z)=\operatorname{rank}\left[f_{1}(z)\right]+\alpha \cdot f_{2}(z)
$$

where $\alpha$ is the weighting coefficient between the accuracy and the complexity of the network. $\operatorname{rank}\left[f_{l}(z)\right]$ is the rank value of $f_{1}(z)$, which defined as:

$$
\operatorname{rank}\left[f_{1}(z)\right]= \begin{cases}1 & f_{1}(z) \leq \varepsilon \\ 2 & f_{1}(z)>\varepsilon\end{cases}
$$


where $\varepsilon$ is the convergence threshold of the L-M BP.

From the above analysis, it can be seen that selection of $\alpha$ is a key question for fitness function. Considering that accuracy of the network is more important than taking the consideration of complexity of the network. Hence the weighting coefficient $\alpha$ could be derived as the following steps:

Let $N_{h}$ be the possible maximum number of hidden neurons and $P$ be the population of the structure-GA loop, then:

$$
f_{2}(z) \leq N_{h}, \quad \forall z \in P
$$

Consider that there is at least one structure of neural networks could finish the training process successfully, which means $\exists z_{i} \in P$, satisfy $f_{l}\left(z_{i}\right) \leq \varepsilon$ and $\operatorname{rank}\left[f_{l}\left(z_{l}\right)\right]=1$. At the same time, suppose $z_{j} \in P$ is failed in convergence, which means $f_{l}\left(z_{j}\right)>\varepsilon$ and $\operatorname{rank}\left[f_{l}\left(z_{j}\right)\right]=2$, then:

$$
\begin{aligned}
& f\left(z_{j}\right)>f\left(z_{i}\right) \\
\Rightarrow & f_{1}\left(z_{j}\right)+\alpha \cdot f_{2}\left(z_{j}\right)>f_{1}\left(z_{l}\right)+\alpha \cdot f_{2}\left(z_{l}\right) \\
\Rightarrow & \alpha<\frac{1}{f_{2}\left(z_{l}\right)-f_{2}\left(z_{j}\right)} \\
\Rightarrow & \alpha<\frac{1}{N_{h}}
\end{aligned}
$$

According this principle to construct fitness function of structure-GA loop, we could get the simplest neural network structure on the basis that the accuracy is guaranteed.

At the beginning of the structure-GA loop, the population is initialized randomly and evaluated using the fitness function. Then we use one-point crossover and simple mutation to generate the new individuals. Crossover operator provides a mechanism for chromosomes to mix and match attributes through random processes. Mutation is a random alteration of some gene values in a chromosome. Every gene in each chromosome is a candidate for mutation. The selection of crossover and mutation is determined by the crossover and mutation rate respectively.

Once the new individuals have been generated, the offspring is selected by the selection scheme. The selection scheme for structure-GA loop is roulette selection method, which selects the offspring according to the fitness. The larger the fitness value, the more opportunities the parents could be selected. After the selection, a new iteration will be carried out until there is no improvement of the best chromosome for several generations.

It can be seen that the global optimal structure of the neural network has been found after structure-GA loop is performed. This provides mathematical support for ANN structure selection, which reduces the enormous search spaces in neural network learning and then improve the system performance.

\section{GA loop for connection weights optimization}

Once the optimal structure of the neural network has been found, it is fed to the connection-GA loop, which has the task of further global optimizing the weights and biases of the neural network with the optimal structure over the training sample set.

The connection-GA loop operates on a chromosome of real-value genes, which, for a given structure, codifies the weights and biases of the neural network directly. Suppose the NN with the optimal structure has $n_{i}$ input neurons, $n_{h}$ hidden neurons and $n_{0}$ output neurons. The weights from input layer to the hidden layer are $\left[w_{11}^{(1)}, w_{12}^{(1)}, \cdots w_{n_{j} n_{h}}^{(1)}\right]^{T}$. Likewise $\left[w_{11}^{(2)}, w_{12}^{(2)}, \cdots w_{n_{k} n_{o}}^{(2)}\right]^{T}$ are the weights from hidden layer to output layer. The chromosome $x$ of the connectionGA loop is $\left[w_{11}^{(1)}, w_{12}^{(1)}, \cdots w_{n_{n_{k}}}^{(1)}, w_{11}^{(2)}, w_{12}^{(2)}, \cdots w_{n_{k} n_{o}}^{(2)}, b^{(1)}, b^{(2)}\right]$.

The fitness function of the connection-GA loop is evaluated with reference to the whole training set in two ways. The first one is the mean squared error between the desired and calculated output of the neural network, which is expressed as $\phi_{1}(x)$ :

$$
\phi_{1}(x)=\frac{1}{N} \sum_{t=1}^{N}(d(t)-y(p, t))^{2}
$$

where the meaning of the parameters is same with (5) though the optimized object is different.

The other way of the fitness function $\phi_{2}(x)$ is the total number of wrong winners in the outputs. Without this item, it is possible for the output to get the wrong winners although the mean squared error has satisfied the convergence condition. So the fitness function of connection-GA loop could be written as:

$$
\phi(x)=\phi_{1}(x)+\phi_{2}(x)
$$

Equal weightings are used for $\phi_{1}(x)$ and $\phi_{2}(x)$ for their similar significance in fault diagnosis.

Because the representation scheme of connection-GA loop is the real-value method, we select arithmetic crossover and non-uniform mutation as the genetic operators. Arithmetic crossover is defined as a linear combination of two selected parents. For example $x_{p}^{l}$ and $x_{q}^{l}$, two parents in $l^{\text {th }}$ generation of connection GA loop, are to be crossed, the resulting offspring are as follows:

$$
\begin{aligned}
& x_{p}^{l+1}=\lambda \cdot x_{p}^{l}+(1-\lambda) \cdot x_{q}^{l} \\
& x_{q}^{l+1}=\lambda \cdot x_{q}^{l}+(1-\lambda) \cdot x_{p}^{l}
\end{aligned}
$$

where $x_{p}^{l+1}$ and $x_{q}^{f+1}$ are two offspring and $\lambda$ is a constant parameter of arithmetic crossover.

The non-uniform mutation operator is defined as follows: If $x_{p}^{\prime}=\left[w_{11}^{(1)}, w_{12}^{(1)}, \cdots w_{n_{i} n_{k}}^{(1)}, w_{11}^{(2)}, w_{12}^{(2)}, \cdots w_{n_{h} n_{0}}^{(2)}, b^{(1)}, b^{(2)}\right]_{p}^{l}$ is the $p^{\text {th }}$ chromosome in the $l^{\text {th }}$ generation of the connection GA loop, in which the element $\left(w_{i j}^{(2)}\right)_{p}^{l}$ is selected for this 
mutation (domain of $\left(w_{i j}^{(2)}\right)_{p}^{l}$ is $\left[a_{i j}^{(2)}, b_{i j}^{(2)}\right]$ ), the offspring is $x_{p}^{l+1}=\left[w_{11}^{(1)}, w_{12}^{(1)}, \cdots w_{n, n_{k}}^{(1)}, w_{11}^{(2)}, w_{12}^{(2)}, \cdots w_{i j}^{(2), \text { new }}, \cdots w_{n_{b} n_{o}}^{(2)}, b^{(1)}, b^{(2)}\right]_{p}$ and $\left(w_{j j}^{(2), n e w}\right)_{p}^{l+1}$ could be obtained by:

$$
w_{i j}^{(2), n e w}=\left\{\begin{array}{l}
w_{i j}^{(2)}+\Delta\left(l, a_{i j}^{(2)}-w_{i j}^{(2)}\right) \text { if a random digit is } 0 \\
w_{i j}^{(2)}-\Delta\left(l, w_{i j}^{(2)}-b_{i j}^{(2)}\right) \text { if a random digit is } 1
\end{array}\right.
$$

where the function $\Delta(l, u)$ returns a value in the range $[0, u]$, which defined as:

$$
\Delta(l, u)=u \cdot\left(1-r^{\left(1-\frac{l}{L}\right)^{\circ}}\right)
$$

where $r$ is a random number from $[0,1] ; L$ is the maximal generation number of the connection-GA loop and $c$ is a system parameter determining the degree of non-uniformity. In addition, the selection scheme of connection-GA loop is roulette method too.

GA is a global optimization algorithm and has no limitation on problem complexity. Therefore using connection-GA loop, instead of BP, to tune the weights and biases of the neural network, the global optimum of the neural network solution could be found and the discontinuous connection weights can be handled. We can see the structureGA loop and the connection-GA loop can jointly search the global optimal NN structure and parameters for fault diagnosis. The computer flow chart of the GA based neural network for fault diagnosis is shown in Fig. 2.

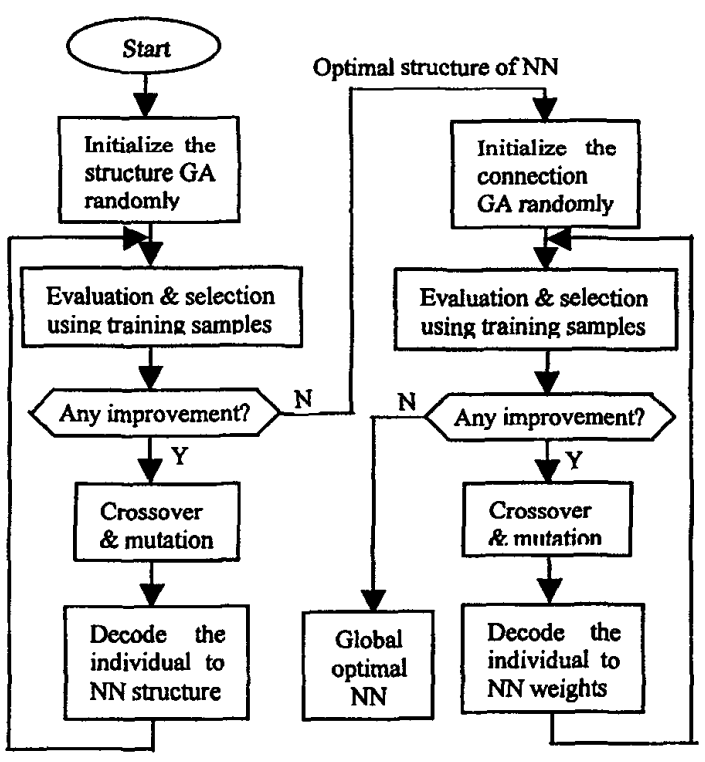

Fig. 2 The computer flow chart of GA based NN

\section{COMPUTER TEST RESULTS}

A simple 4-bus power system ${ }^{[11]}$ is used as the test system (Fig. 3), in which there are 9 protected components: 4 buses,
1 transformer and 4 transmission lines. The protection relay system considered in the computer test is a simplified system, which includes transmission lines main protection (MLP) and backup protection $(B L P)$, main protection for buses $(M B P)$ and the transformer $(M T P)$.

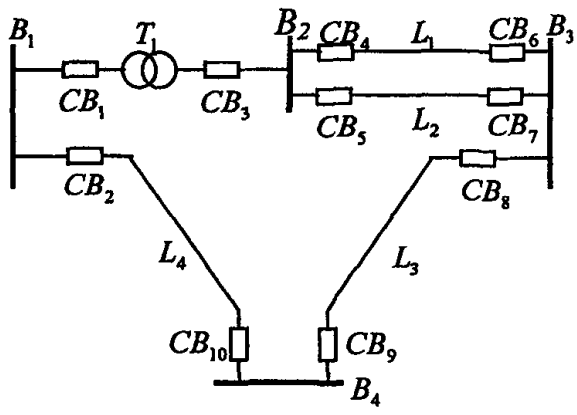

Fig. 3 Test power system diagram

In computer tests, forty typical fault scenarios $(N=40)$ are worked out to constitute the training sample set. For each fault scenario, the states of all relays and circuit breakers $(0$ or 1) are taken as the NN inputs $\left(n_{i}=33\right)$. The states of the 9 system components ( 4 buses, 1 transformer and 4 lines) are the outputs $\left(n_{o}=9\right)$. If a certain output approaches to 1 , then the corresponding component is considered in fault.

Based on the given training samples, run structure-GA loop to find the optimal structure of the neural network for fault diagnosis. The parameters of the structure-GA loop are as follows: each generation has 10 individuals and each individual has 6 genes; weighting coefficient $\alpha=0.03$; the one-point crossover rate is 0.4 ; the simple mutation rate is 0.008 . After 12 generations, the structure-GA loop converges to the optimal structure, which has 7 hidden neurons and their activation function is "logsig". The convergence curve is shown in Fig. 4.

The optimal NN structure is fed to connection-GA loop to further tune its weights and biases. We select the parameters of connection-GA loop as follows: there are 50 individuals in each generation; the arithmetic crossover rate is 0.40 ; the non-uniform mutation rate is 0.003 . Then after 1500 generations, connection-GA converged. The convergence curve is shown in Fig. 5.

In addition, in order to prove that the obtained neural network structure is global optimal, we compare the results of the connection-GA loop with different number of hidden neurons (Fig. 6). Besides the optimal neural network structure of 7 hidden neurons, 5 and 9 hidden neurons cases are considered. When the numbers of hidden neurons is 5 , which is less than the optimal value, the connection-GA loop saturate early and could not improve further, therefore it is poor in output accuracy. On the other hand, when the numbers of hidden neurons is larger than the optimal value, though the connection-GA loop could converge successfully, the structure of the neural network is more complex. This is 
not our desired situation. So the computer simulation results prove that the optimal neural network structure could be found using the structure-GA loop.

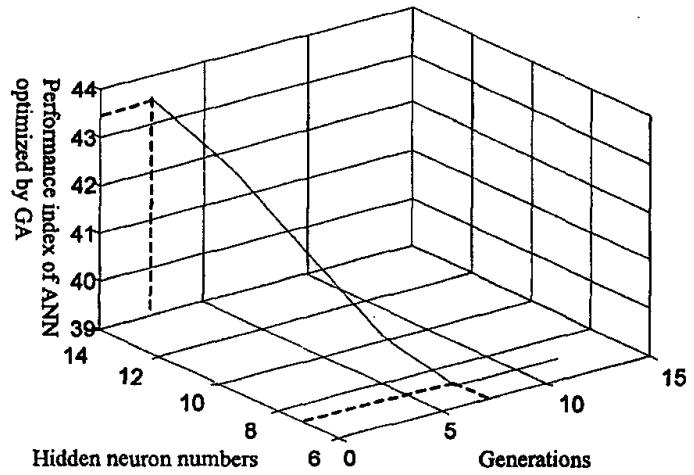

Fig. 4 Convergence curve of structure-GA loop

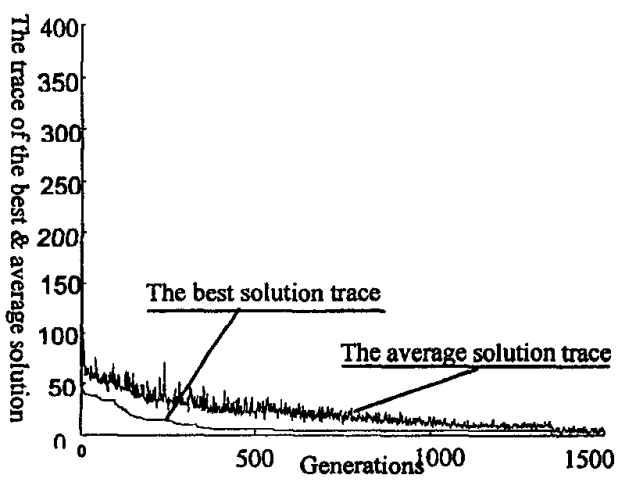

Fig. 5 Convergence curve of connection-GA loop

In the same time, we apply BP learning algorithm to train the neural network with the optimal structure using the same training sample set. Its convergence curve is shown in Fig. 7. From Fig. 6 and Fig. 7, it can be seen that connection-GA loop takes much less generations to converge than that of the BP neural network.

In order to examine the generalization capability of the designed NN we select other 12 fault scenarios not existing in the training set for testing. All of them are severe cases with up to 2 mal-operations of relays and circuit breakers or up to 2 simultaneous faults. One example is given as follows. Suppose line 4 has a fault. If the main protection of $C B_{10}$ operated correctly and trip $C B_{l o}$ instantly, while the main protection of $C B_{2}$ refused to operate and the back-up protection of $C B_{3}$ trip $C B_{3}$ after a time delay. When these alarms are feed to the input of the neural network, the outputs are as follows: $[0.1841,-0.0803,0.1116,-0.0902,0.0798$, $0.0398,0.0214,0.0175,0.8398]^{\mathrm{T}}$. Then we could get the correct diagnosis result that line 4 has a fault. The similar results are obtained in other test cases. So the test results prove that GA based neural network fault diagnosis system could implement fault diagnosis accurately.
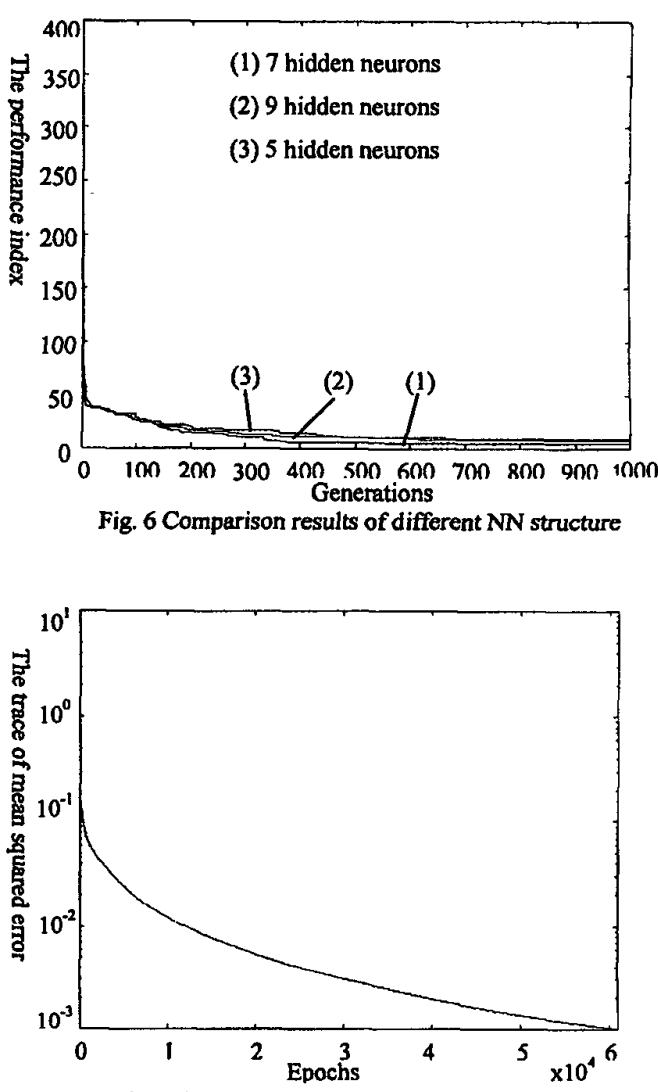

Fig. 7 Convergence curve of BP neural network

\section{CONCLUSION}

In this paper, a novel Genetic Algorithm (GA) based neural network for fault diagnosis in power systems is suggested, which adopts three-layer feed-forward neural network. Dual GA loops are applied in order to optimize the neural network topology and the connection weights. The first GA-loop is for structure optimization and the second one for connection weight optimization. Jointly they search the global optimal neural network solution for fault diagnosis. The formulation and the corresponding computer flow chart have been presented in detail. Computer test results for fault diagnosis in test power system indicate that the proposed GAbased neural network fault diagnosis system works well and is superior as compared with the conventional BackPropagation (BP) neural network.

\section{ACKNOWLEDGMENTS}

The research is supported by National Key Basic Research Special Fund (No. G1998020305). 


\section{REFERENCES}

[1] C. Fukui and J. Kawakami, "An expert system for fault section estimation using information from protective relays and circuit breakers", IEEE Trans. Power Delivery, Vol. 1, No. 4, October 1986, pp. 83-90

[2] M. Ernesto Vazquez, L. Oscar, M. Chacon, J. Hector and F. Altuve, “ An on line expert system for fault section diagnosis in power system", IEEE Trans. Power Systems, Vol.12, No.1, February 1997, pp.357-362.

[3] Hyun-Joon Cho and Jong Keun Park, "An expert system for fault section diagnosis of power systems using fuzzy relations", IEEE Trans. Power Systems, Vol.12, No.1, February 1997, pp.342-348.

[4] C.S. Chang, J.M. Chen, D. Srinivasan, F.S. Wen and A.C. Liew, "Fuzzy logic approach in power system fault section identification", IEE Proceedings: Generation, Transmission and Distribution, Vol.144, No.5, September 1997, pp.406-414.

[5] T. Oyama, "Fault section estimation in power system using Boltzmann machine", Proceedings of the Second International Forum on Application of Neural Networks to Power Systems (ANNPS'93), pp 3-8.

[6] C.S. Chang, L. Tian and F.S. Wen, "A new approach to fault section estimation in power systems using ant system", Electric Power System Research, 1999(41), pp. 63-70

[7] Young Moon Park and Gwang-Won Kim, " A logic based expert system (LBES) for fault diagnosis of power system", IEEE Trans. Power Systems, Vol.12, No.1, February 1997, pp.363-369.

[8] T.S. Sidhu, O. Cruder and G.J. Huff, “ An abductive inference technique for fault diagnosis in electrical power transmission networks", IEEE Trans. Power Delivery, Vol.12, No.1, January 1997, pp.515-522.

[9] V. Navarro, A.L. da Silva, L.A.V. de Carvalho and G. Zaverucha, "Artificial neural networks for power systems diagnosis", 1994 IEEE International Conference on Neural Networks, 1994, pp. 3738-3743.

[10] Y.M. Sun, H. Jiang and D. Wang "Fault synthetic recognition for an EHV transmission line using a group of neural networks with a timespace property", IEE Proceedings: Generation, Transmission and Distribution, Vol.145, No.3, May 1998, pp. 265-270.

[11] Z.E. Aygen, S. Seker, M. Bagriyanik, F.G. Bagriyanik and E. Ayaz, "Fault section estimation in electrical power systems using artificia neural network approach", 1999 IEEE Transmission and Distribution Conference, 1999 , pp. 466-469.

[12] D.E. Rumelhart, G.E. Hinton and R.J. Williams, "Learning internal representations by error propagation", Parallel Distributed Processing: Explorations in the Microstrcture of Cognition, ed. D.E. Rumelhart and J.L. McClelland, MTT Press, Cambridge, MA, pp. 318-362.

[13] D.W. Marquardt, “ An algorithm for least-squares estimation of nonlinear parameters", J. Soc. Ind. Appl. Math., Vol.11, 1963, pp. 431441
[14] Zbigniew Michalewicz, Genetic Algorithms + Data Structures= Evolution Programs, Springer, 1992

[15] M. Borairi and H. Wang, "Actuator and sensor fault diagnosis of nonlinear dynamic systems via genetic neural networks and adaptive parameter estimation technique", Proceedings of the 1998 IEEE International Conference on Control Applications, Italy 1998, pp. 278282

[16] Chih-hsien Kung, Michael J. Devaney, Chung-ming Huang and Chihming Kung, "An adaptive power system load forecasting scheme usin a genetic algorithm embedded neural network", Proceedings of the 1998 IEEE Instrumentation and Measurement Technology Conference, USA 1998, pp. 308-311

\section{VII.BIOGRAPHIES}

T.S. Bi received her B. Eng, and M. Eng degrees from North China Electric Power University, P. R. China, in 1994 and 1997 respectively. She is currently a Ph. D. candidature at the Dept. of E.E.E., the University of Hong Kong. Her research area includes power system operation, protective relay and the application of artificial intelligence in power systems.

Y. X. Ni received her B. Eng., M. Eng., and Dr. Eng. from Electrical Engineering Department, Tsinghua University, P. R China in 1968, 198 and 1983 respectively. Her research interest is in power system modeling, simulation, stability and control, and power electronics applications in power systems. She was a professor of Tsinghua University and is now with the University of Hong Kong.

C.M. Shen received his B. Sc. (Eng.) and M. Sc. (Eng.) degrees in EE from the University of Hong Kong (HKU) in 1963 and 1965 respectively and his Ph.D. degree in EE from Queen Mary College, University of London in 1969. Since then he has joined the HKU. His research interest is in Load flow, fault analysis, state estimation and stability and control of power systems. He is an executive committee member of the Specialized Section in Power, IEE Hong Kong Center.

F.F. Wu joined the University of Hong Kong as Chair professor of Electrical Engineering in September 1995 and is now Pro-vice-chancellor of HKU. Prior to that, he was Professor and Vice Chairman, Department of Electrical Engineering and Computer Sciences, University of California, Berkeley, the same institute where he received his Ph.D. degree. His research interests are in power system planning and operation, including economics and reliability in system planning, real-time security assessment, and design of energy management systems and distribution automation. Recently he has been involved in the design of industry restructuring and electricity pricing. 